\title{
Synthesis of gold-poly (dimethylsiloxane) nanocomposite through a polymer-mediated silver/gold galvanic replacement reaction
}

\author{
Pooja Devi ${ }^{\dagger}$, Simona Badilescu*, ${ }^{\star}$, Muthukumaran Packirisamy ${ }^{\ddagger}$ and Pethaiyan Jeevanandam ${ }^{\dagger}$, \\ www.goldbulletin.org
}

\begin{abstract}
Silver-poly (dimethylsiloxane) nanocomposite films are prepared by an in-situ synthesis by incubating the polymer film in a silver nitrate aqueous solution and using the reducing properties of the polymer's curing agent. Silver nanoparticles concentrated in the surface layer of the silver-poly (dimethylsiloxane) nanocomposite are further replaced by gold through a galvanic replacement reaction at the poly (dimethylsiloxane)/gold salt solution interface. It is demonstrated that, as a result of the galvanic replacement, the quasi-spherical silver nanoparticles uniformly distributed on the surface of the polymer are transformed into ribbon-like elongated gold aggregates. The formation of core-shell Ag-Au nanoparticles as an intermediate state of the low-temperature galvanic replacement is evidenced through microscopic and spectral methods. The collapse of this structure, in a later stage of the replacement, leads to the formation of elongated gold aggregates. The mechanism of the galvanic replacement under different conditions is investigated by closely following the evolution of the morphology, composition, and optical properties of the metal nanoparticles.
\end{abstract}

\footnotetext{
Optical Bio-Micro Systems Laboratory, Department of Mechanical and Industrial Engineering, Concordia University,1455 de Maisonneuve Blvd. West, Montreal (Quebec), Canada H3G 1 M8

Centre for Nanotechnology and "Department of Chemistry, Indian Institute of Technology, Roorkee (Uttarakhand), India 247667

* To whom correspondence should be addressed, Fax: (514)8483175, Tel: (514)8482424 ext. 7098, Email: sbadiles@ece.concordia.ca
}

\section{Introduction}

Synthesis of various noble metal nanostructures has been an active area for decades, because of their interesting properties and their importance to a variety of applications such as photonics, SERS, optoelectronics, catalysis and chemical \& biological sensing [1-4]. By controlling the size, shape, and composition (in the case of bimetallic nanoparticles), their properties can be tailored. Particularly, for $\mathrm{Au}$ and $\mathrm{Ag}$, the resonance frequency of surface plasmon (SPR) can be tuned by adjusting the size and morphology or by alloying. In some cases, the SPR band could be shifted to the nearinfrared region, a region of particular interest for biomedical applications such as for example, cancer hyperthermia $[5,6]$.

Xia et al. has developed a method to prepare hollow nanostructures with controlled pore volume and wall thickness, by using the galvanic replacement reaction carried out in aqueous solutions [7-9]. This reaction is based on the reduction potential difference between silver and gold ( $\mathrm{AuCl}_{4}^{-} / \mathrm{Au}$ is $0.99 \mathrm{~V}$, which is higher than $\mathrm{Ag}^{+} / \mathrm{Ag}=0.8 \mathrm{~V}$ ) that allows the oxidation of $\mathrm{Ag}$ nanostructures by the solution of the gold salt. The gold that results in the replacement reaction grows on the silver template adopting its morphology and forms a thin shell around it. Hollow structures are formed by the further oxidative dissolution of the core. In some cases, under appropriate conditions, the gold could alloy with the un-reacted silver [10]. The galvanic replacement procedure was extended to other noble metals ( $\mathrm{Pt}$ and $\mathrm{Pd}$ ) by using a silver template and the corresponding salts. It has been demonstrated that the size and shape of the initial $\mathrm{Ag}$ nanoparticles determine the morphology of the structures generated by the replacement reaction. Recently, the galvanic replacement reaction was studied in a highly viscous ionic liquid environment and the transformation of quasi-spherical $\mathrm{Ag}$ 
nanoparticles into dendritic flat Au-Ag nanostructures has been demonstrated [11]. Ag@Au core-shell nanostructures were also prepared by using a hexagonal shape $\mathrm{Ag}$ template and performing the galvanic replacement reaction between $\mathrm{Ag}$ and $\mathrm{HAuCl}_{4}$ in the presence of poly(vinyl pyrrolidone) [12].

Poly (dimethylsiloxane) (PDMS) is a polymer used for the fabrication of microfluidic devices. It has received the most attention due to its ease of preparation, low cost, good transparency and non-toxicity to biomolecules. Due to these properties, PDMS can be used directly for cell culturing $[13,14]$. In addition, PDMS has a low glass transition temperature $\left(T_{q}\right)$, excellent flexibility, high thermal and oxidative stability, good hemo- and biocompatibility [15], properties that make it a very attractive polymer host for the fabrication of nanocomposites [16]. After crosslinking, it becomes an elastomeric material with a low Young's modulus of $\sim 750 \mathrm{kP}$ which enables it to form reversible seals and it can be molded into functional devices. Gold (silver) - PDMS nanocomposites are important functional materials with interesting potential applications as sensors, filters, low refractive index materials and thermochromic materials $[17,18]$. In a previous work, we have prepared Ag - PDMS and Au - PDMS nanocomposites for biosensing applications, by incubating PDMS films prepared with a high concentration of curing agent, for at least 18 hours in an silver nitrate $\left(\mathrm{AgNO}_{3}\right)$ or chloroauric acid $\left(\mathrm{HAuCl}_{4}\right)$ aqueous solution at room temperature [19]. Our results showed that the metal nanoparticles formed by reducing the corresponding salts by the curing agent, are concentrated on the surface of the polymer film and can be used for biosensing. The amount of metal nanoparticles on the surface depends on both, the concentration of the salt solution and time of incubation, that is, the time the polymer and the solution are in contact. The gold and silver nanoparticles prepared through this procedure are quasi-spherical and their size was found to be around 100-150 nm. Any attempt to prepare AuAg alloys by co-reduction of chloroauric acid and silver nitrate by using the same procedure, failed. The UV-Visible spectra showed that, invariably, after incubating for several hours the polymer film in a solution containing both gold and silver salts, gold alone was found on the surface of the polymer. This intriguing result suggests that silver nanoparticles, if formed in the process, are transformed immediately to gold by a galvanic replacement reaction. Preliminary spectral results have shown that, when a Ag-PDMS composite film is incubated in a $\mathrm{HAuCl}_{4}$ solution, Ag will be gradually replaced by gold, the reaction proceeding through several intermediate states. The aim of this work is to investigate in depth the $\mathrm{Ag} / \mathrm{Au}$ galvanic replacement reaction at the PDMS/aqueous solution interface and to obtain further insights about the mechanisms responsible for the morphological and spectral changes involved in this process.

\section{Experimental section}

\section{Materials}

All chemical were used as received, without any further purification: Sylgard ${ }^{\circledR} 184$ elastomer kit for PDMS fabrication (Dow Corning Corporation), $\mathrm{AgNO}_{3}$ (Sigma Aldrich) and $\mathrm{HAuCl}_{4} \cdot 3 \mathrm{H}_{2} \mathrm{O}$ (Alfa Aesar). Deionized water obtained from NANOpure ultrapure water system (Barnstead) was used in all the experiments.

\section{Preparation procedure}

Poly (dimethylsiloxane) was fabricated by mixing the prepolymer and curing agent in a ratio of $4: 1$ ( $\eta=$ 0.25), followed by evacuation for 15 minutes to remove air bubbles. The degassed assembly was kept in the oven overnight for curing at $60^{\circ} \mathrm{C}$. Samples of size $3 \times 1 \times 0.1 \mathrm{~cm}^{3}$ were cut from the cured PDMS. PDMS samples were incubated for different times (3-48h) in silver nitrate solution. The samples obtained after incubation in $15 \mathrm{mM} \mathrm{AgNO}_{3}$ for 22 hours were further immersed in $0.5 \mathrm{mM}$ $\mathrm{HAuCl}_{4} \cdot 3 \mathrm{H}_{2} \mathrm{O}$ solution at different temperatures $\left(0^{\circ} \mathrm{C}\right.$, $\left.20^{\circ} \mathrm{C}, 40^{\circ} \mathrm{C}\right)$. Incubation was done for times ranging from a couple of seconds to several hours.

\section{Instrumentation}

A Hitachi S-4700 FEG-SEM was employed for imaging the composite films. EDX analysis was done by Hitachi S-3000N VP-SEM equipped with an X-Ray analyzer (Link manufactured by Oxford Company). The $M \alpha$ line of $A u$ and $L \alpha$ line of $A g$ were selected. For SEM imaging, samples were coated by Au/Pd for one minute using the Hummer VI sputtering system. Quantitative elemental analysis was also done by A ESCALAB 3 MKII X-ray photoelectron spectroscopy (XPS) system manufactured by Kratos. The spectra were recorded by using a PerkinElmer ${ }^{\circledR}$ Lambda650 UV-Visible spectrophotometer in the wavelength range, $350-850 \mathrm{~nm}$.

\section{Results and discussion}

The Ag/Au galvanic replacement reaction in water and organic solvents is usually described by 
the stoichiometric oxidation of $\mathrm{Ag}$ atoms by the $\left[\mathrm{AuCl}_{4}\right]$ ] ions [20]:

$$
3 \mathrm{Ag}(\mathrm{s})+\mathrm{AuCl}_{4}^{-}(\mathrm{aq}) \rightarrow \mathrm{Au}(\mathrm{s})+3 \mathrm{Ag}^{+}(\mathrm{aq})+4 \mathrm{Cl}(\mathrm{aq})
$$

When the replacement reaction is performed in an aqueous solution at high temperature, the transformation from Ag to Au nanoparticles involves a number of processes including dissolution of the silver template with generation of gold atoms, deposition of gold on the surface of the template, growth of the gold nanoshells around the dissolving silver template, diffusion of $\mathrm{Ag}$ into the gold shell and formation of Au-Ag alloys and, finally, a dealloying process accompanied by a morphological transformation [8]. In this case, as in all previous studies, the reaction is carried out by adding increasing amounts of $\mathrm{HAuCl}_{4}$ solution to the silver nanostructures. In the present work, the galvanic replacement reaction is carried out by increasing gradually the time of incubation of an Ag-PDMS nanocomposite in a $\mathrm{HAuCl}_{4}$ aqueous solution with an adequate concentration and it takes place at the polymer/solution interface.

Figure 1 shows the spectra of the Ag-PDMS prepared by incubating a thick PDMS film in the silver nitrate solution (15 mM) for times ranging from 1 to 48 hours. The figure shows that the absorbance of Ag LSPR

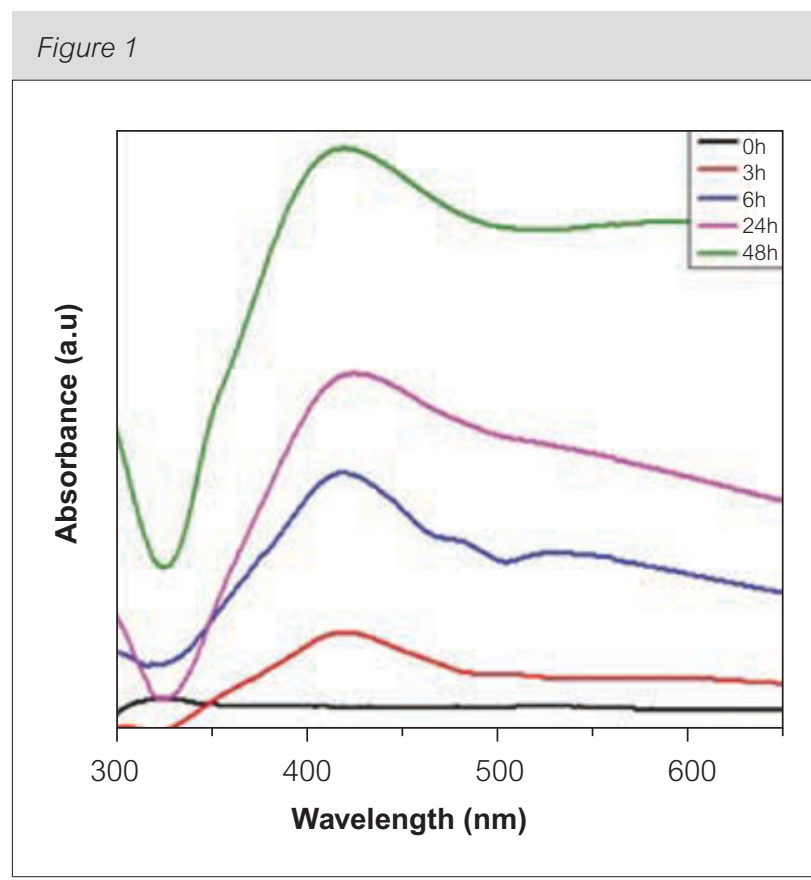

UV-Visible spectra of Ag-PDMS nanocomposite prepared by incubating the PDMS film in a silver nitrate solution (15 mM) for times ranging from 0 to 48 hours band increases with the time of incubation. As shown in our previous work, Ag nanoparticles are formed by reduction of $\mathrm{AgNO}_{3}$ by the curing agent. The curing agent, together with PDMS oligomers, segregated in the surface layer, permits the concentration of the Ag nanoparticles formed by the reduction reaction, in this layer. Ag nanoparticles synthesized in this way are of quasi-spherical morphology with an average diameter of 100-150 nm and, when kept in de-ionized water, the structure is stable for weeks.

Figure 2 shows the SEM images associated with the replacement experiment carried out at room temperature. Figure 2a shows the SEM images of the Ag-PDMS prepared by leaving a thick PDMS film in the $\mathrm{AgNO}_{3}$ solution (15 mM) for 22 hours. Silver nanoparticles and aggregates of $\mathrm{Ag}$ can be seen, aggregation occurring probably during the
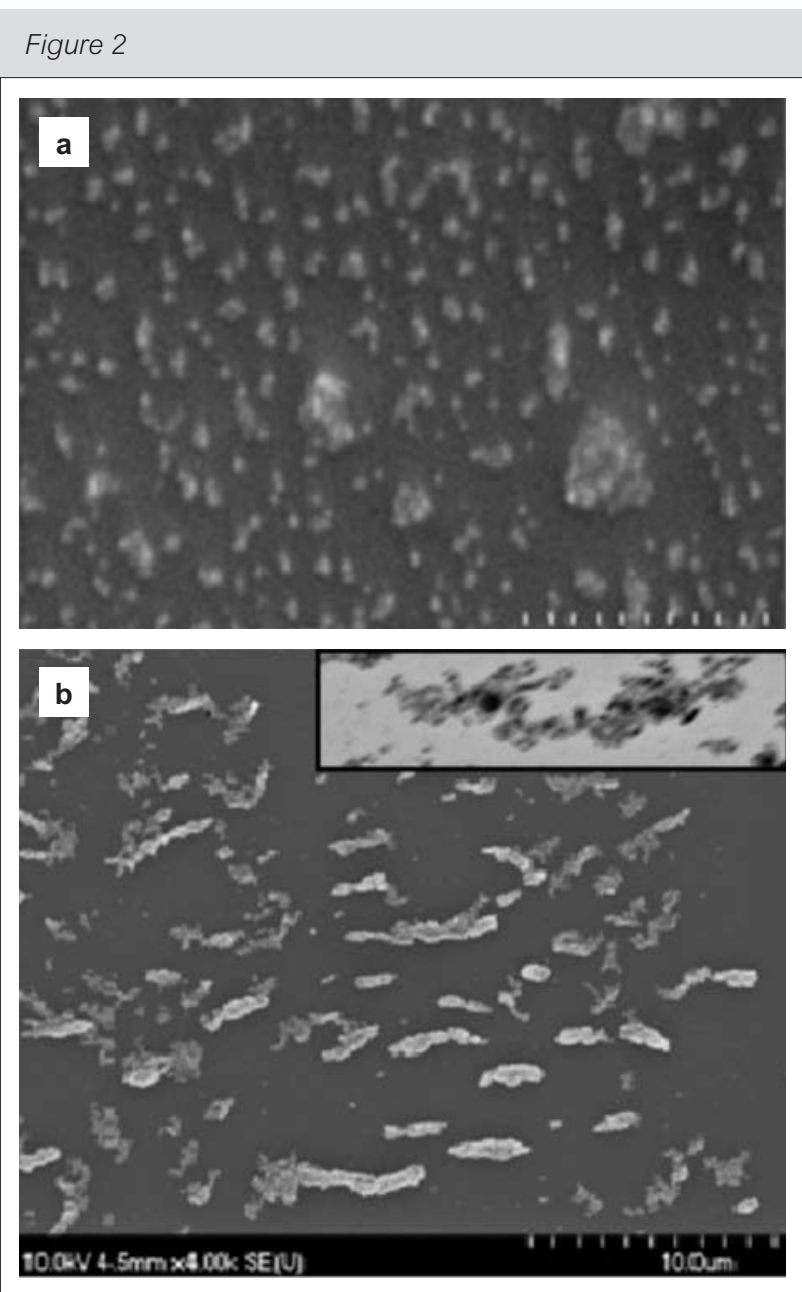

SEM image of Ag-PDMS prepared by keeping the PDMS film in the $\mathrm{AgNO}_{3}$ solution (15 mM) for 22 hours (a) and Au-PDMS prepared by keeping the Ag-PDMS nanocomposite in the $\mathrm{HAuCl}_{4}$ solution for 7 hours. Inset: enlarged image with reversed color for clarity (b) 
reduction reaction. Exposure of Ag-PDMS to the $\mathrm{HAuCl}_{4}$ solution for 7 hours results in the complete replacement of silver by gold (Figure 2b). The image shows aggregated structures, mostly with elongated shapes. The backbones of the aggregates are formed from several rows of spherical particles at which branched structures are attached. Figure 3 shows the spectra corresponding to the evolution with time of the $\mathrm{Ag} / \mathrm{Au}$ replacement reaction. The band at $540 \mathrm{~nm}$ in Figure 3a corresponds to Au, the end product of the replacement reaction under these conditions. In an attempt to capture some of the intermediate states of the replacement reaction, the Ag-PDMS sample was kept in the gold salt solution for different times (few seconds to 10 hours) and the UV-Visible spectra corresponding to the different stages of the replacement reactions carried out at

\section{Figure 3}

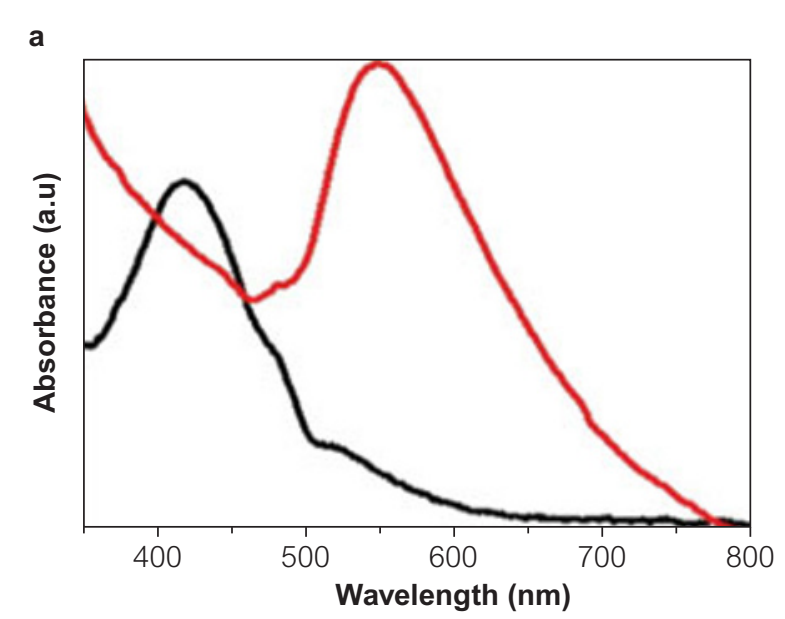

b

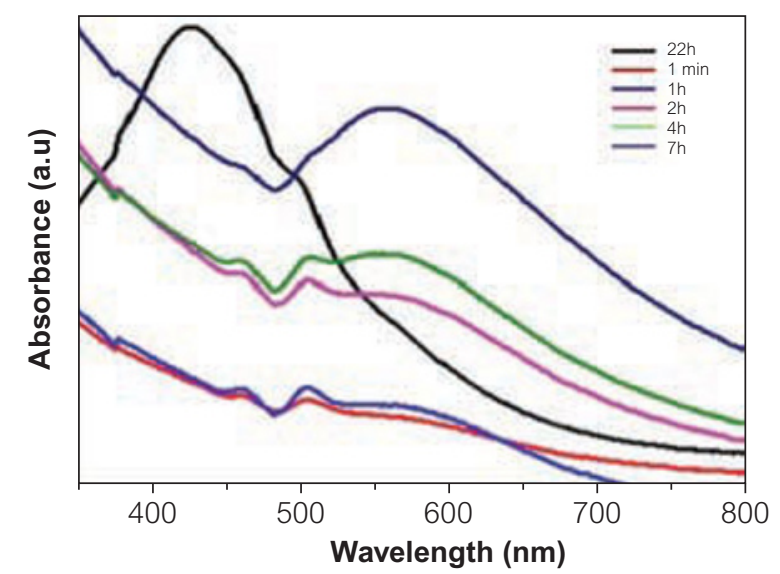

UV-Visible spectrum corresponding to initial (black curve) and final stage (red Curve) of Ag/Au replacement reaction (a) and Spectra corresponding to the evolution with time of the Ag/Au replacement reaction (b) room temperature are shown in Figure 3b. Weak bands at 462 and around $503 \mathrm{~nm}$ can be seen in the spectrum corresponding to a sample dipped into the solution for a very short time (couple of seconds). The spectra show that, immediately upon dipping, there is already some replacement, as a shoulder around $520 \mathrm{~nm}$ can be seen together with the two bands at 462 and $503 \mathrm{~nm}$; these two bands belong to the PDMS curing agent but it is not clear at this point, why the bands due to the curing agent cannot be seen in the spectra of Ag-PDMS and in the end product of the replacement reaction. Their position is the same in all the spectra corresponding to different time of incubation, while the intensity of the Au LSPR band increases with time. After 7 hours, the $\mathrm{Ag} / \mathrm{Au}$ replacement is almost completed and a broad band at $560 \mathrm{~nm}$ shows the exclusive presence of gold nanoparticles. Experiments were performed at $40^{\circ} \mathrm{C}$ as well but the replacement reaction was completed after approximately $15 \mathrm{~min}$ and no intermediate states could be evidenced.

In order to decrease the rate of the replacement and study its mechanism, the replacement reaction was carried out at low temperature $\left(0^{\circ} \mathrm{C}\right)$ and the morphology and spectra of the intermediate states were further investigated. In the very beginning of the replacement reaction, some interesting structures were found as shown in Figure 4. In these images, the dark spots on the silver nanoparticles are gold particles that seem to take the shape of the underlying silver. Figure $4 \mathrm{a}$ is a large scale $(10 \mu \mathrm{m})$ image of the sample right after dipping it in the gold salt solution. It can be clearly seen, the gold that surrounds the silver nanoparticles and aggregates at the very beginning of the replacement reaction. On this large scale image, the particles seem to be arranged along parallel straight lines. This may be the result of some displacement of silver nanoparticles by the solution that withdraws from the sample after removing the sample from the solution. Figure $4 \mathrm{~b}$ and $4 \mathrm{c}$ show the close-up images of core-shell particles that have a quasi-spherical shape (see inset Figure 4b). However, as it can be seen in the figure, particles having other shapes, some of them anisotropic, are present in the sample as well. The spectrum corresponding to the low temperature replacement reaction (Figure 5) shows two broad Ag and Au LSPR bands (initial and end states), together with a broad absorption at higher wavelengths (around $800 \mathrm{~nm}$ ) attributed to the intermediate state that could belong to a core-shell nanoparticle and/or the anisotropic particles seen in the SEM images. 

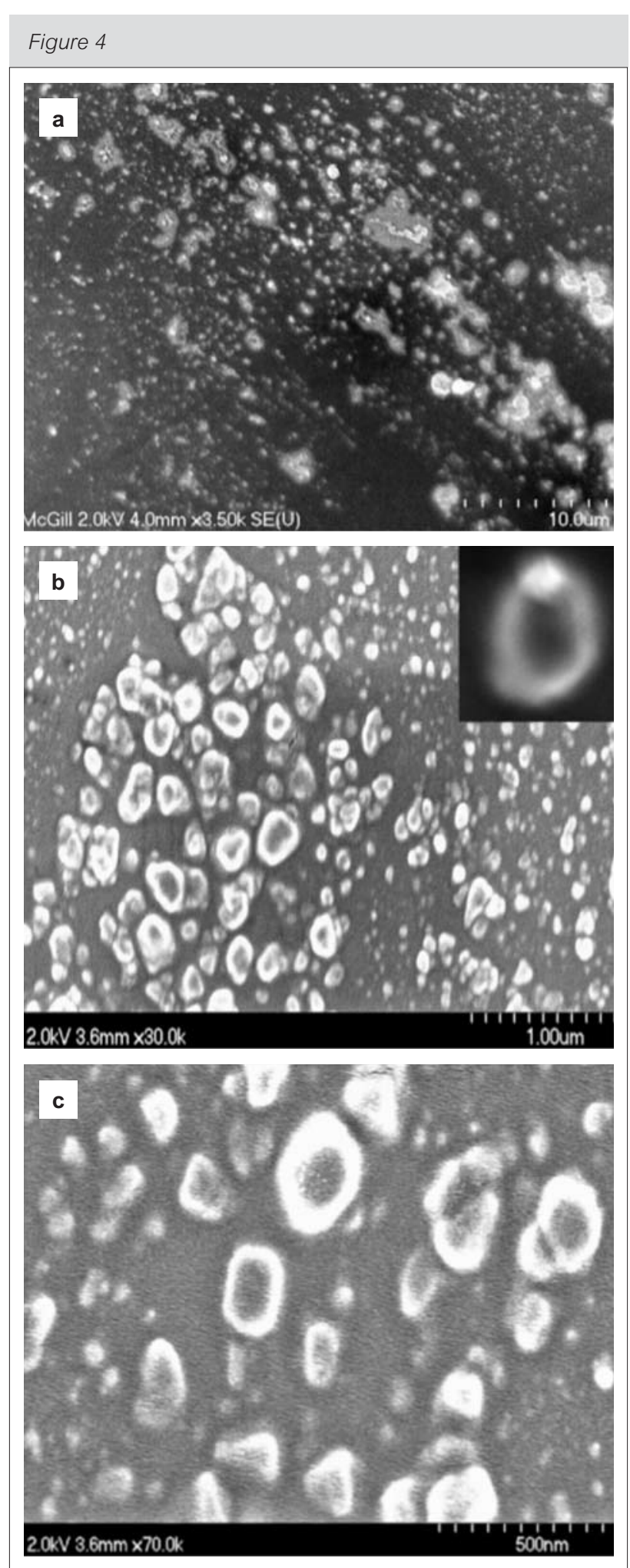

SEM images corresponding to an intermediate state of the replacement reaction at low temperature (a) large scale $(10 \mu \mathrm{m})$ image showing the gold solution patches around the silver nanoparticles and aggregates (b) 1 um scale image showing core-shell structures of various sizes and shapes (c) high-magnification (500 nm) image of structures seen in image (b)

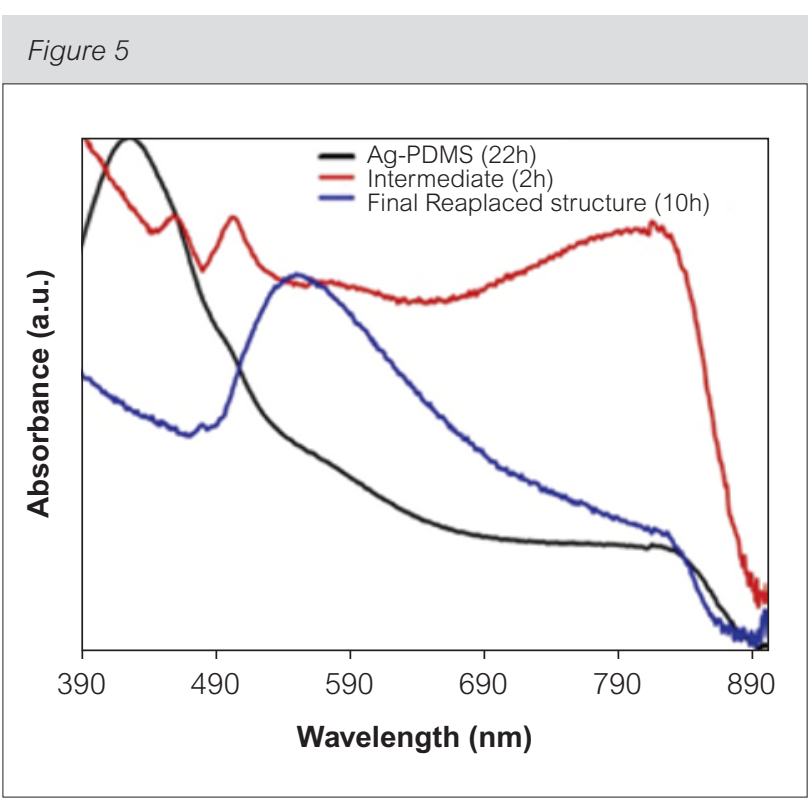

Spectra corresponding to the evolution of the replacement reaction at low temperature

The Au/Ag particles shown in Figure 4 are coreshell particles of a great size and shape diversity illustrated by the broad Ag and Au LSPR bands. No tuning of the Au LSPR band with the time of incubation, that is, with increasing Au concentration can be achieved as in the case of replacement reactions carried out in water at high temperatures. This result can be accounted for by the fact that the replacement reaction proceeds at different rates on different particles because of their diversity in size and shape. At a given time, the replacement can be in an early stage, or a more advanced one, depending on the size and shape of the underlying silver particle. Because of the large size of the original particles (100-150 nm), at some point during the replacement, the core-shell structure will collapse and elongated assemblies of Au nanoparticles are formed as shown in Figure 2b.

The changes in the chemical composition shown in Figure 6 (EDX) and in Table 1 (XPS) are in a good agreement with the previous results. Indeed, Figure $6 \mathrm{~b}$ corresponding to the intermediate state shows the presence of both $\mathrm{Ag}$ and $\mathrm{Au}$ in the sample. This result is confirmed by the XPS data as well. It is interesting to note that some $\mathrm{Ag}$ is still present in the end product of the replacement; however, no Ag band can be seen in the spectra. Silver originates probably, from $\mathrm{AgCl}$, the insoluble product of the replacement which cannot be removed because of its low solubility at room temperature. 


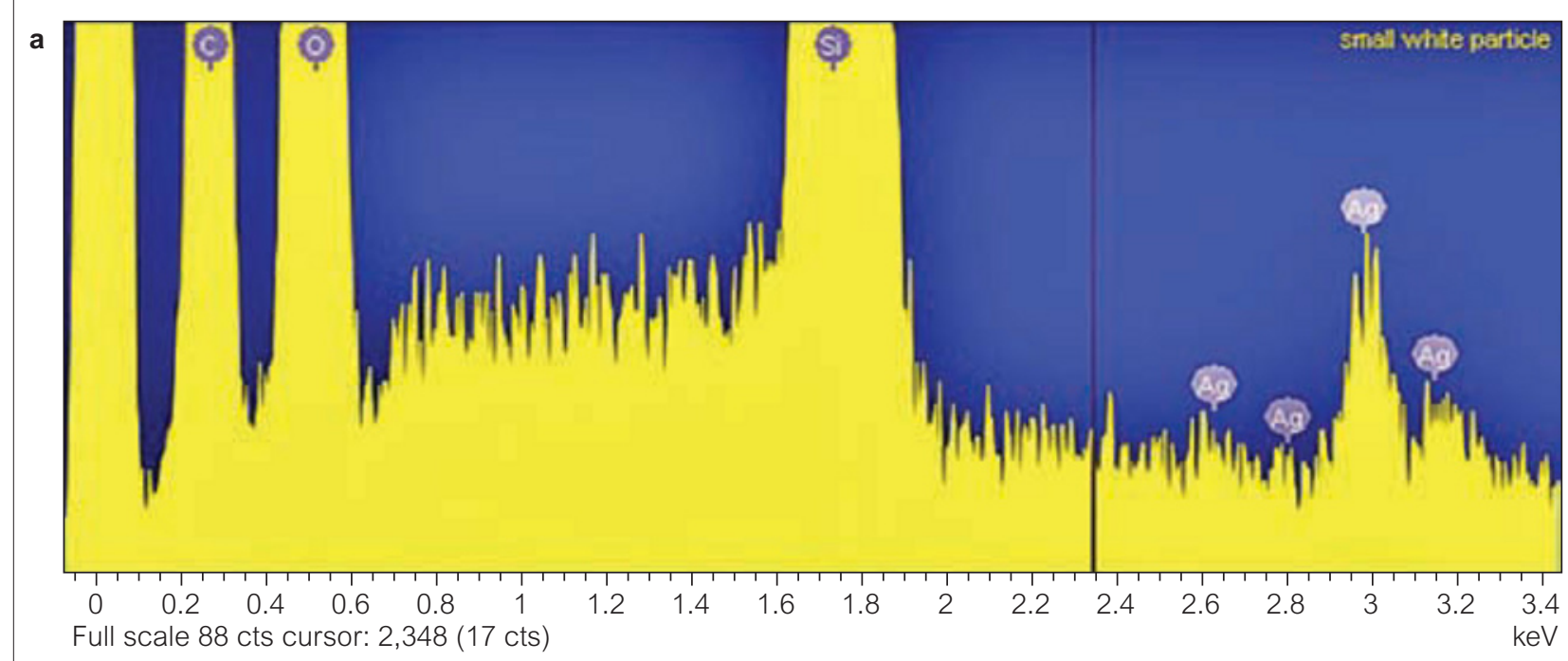

b

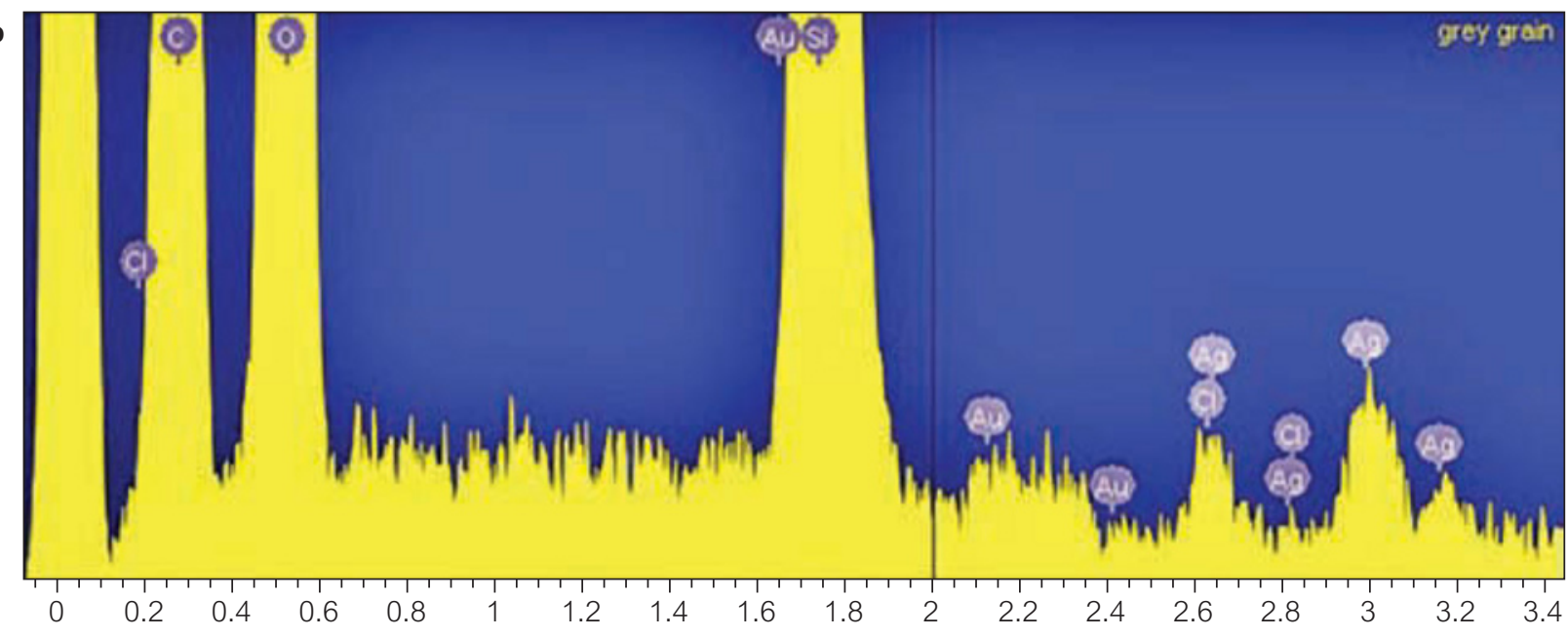

c

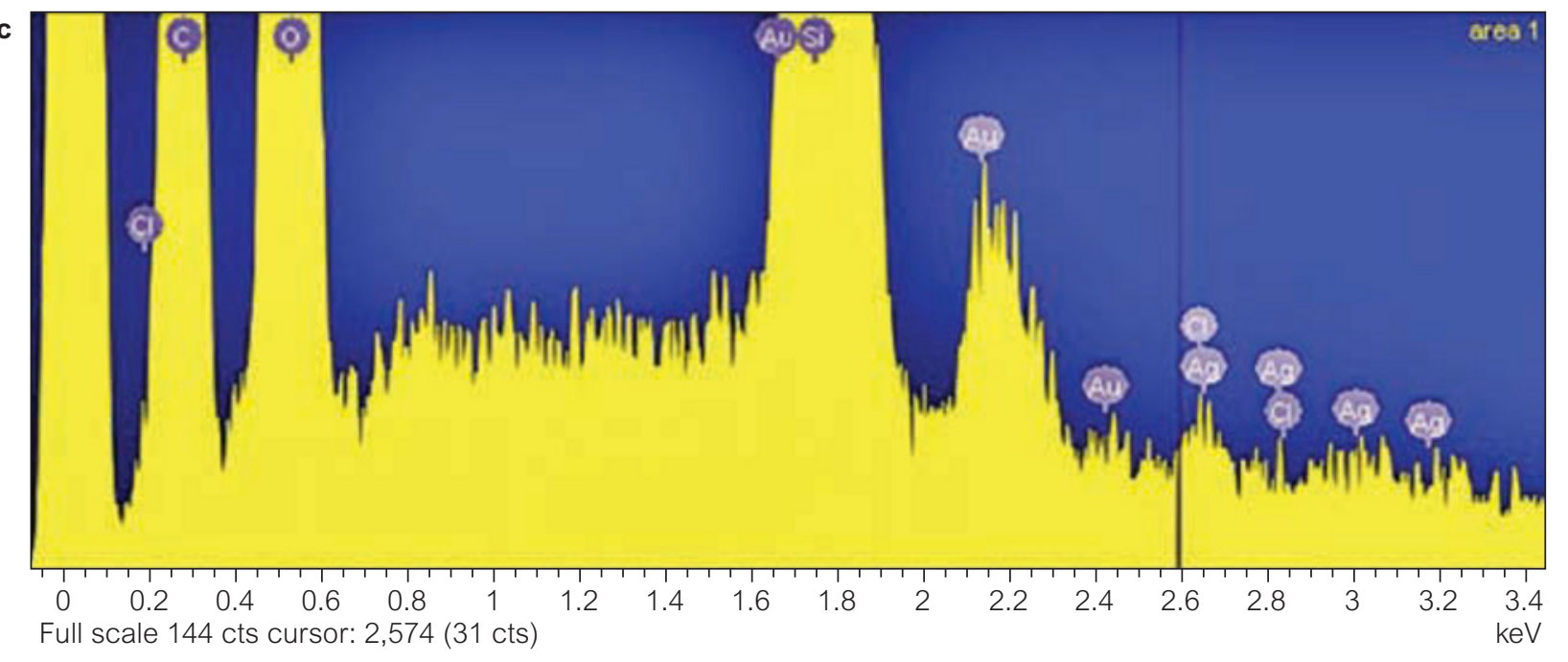

EDX results corresponding to the room temperature galvanic replacement reaction (a) Ag-PDMS nanocomposite (initial state); (b) intermediate state; (c) Au-PDMS composite (final state) 
Table 1: XPS data corresponding to the galvanic replacement reaction

\begin{tabular}{|c|c|c|c|c|c|}
\hline Sample & $\begin{array}{r}\text { Elemental } \\
\text { ID }\end{array}$ & B.E & $\begin{array}{l}\text { Height } \\
\text { CPS }\end{array}$ & SF & $\begin{array}{r}\text { Atomic } \\
\text { Percentage }(\%)\end{array}$ \\
\hline PDMS & $\begin{array}{l}\mathrm{Si} 2 \mathrm{p} \\
\mathrm{C} 1 \mathrm{~s} \\
\mathrm{O} 1 \mathrm{~s}\end{array}$ & $\begin{array}{l}103.05 \\
285.28 \\
533.11\end{array}$ & $\begin{array}{r}41258.12 \\
76309.67 \\
109421.07\end{array}$ & $\begin{array}{l}0.27 \\
0.25 \\
0.66\end{array}$ & $\begin{array}{l}22.25 \\
47.83 \\
29.92\end{array}$ \\
\hline Ag-PDMS & $\begin{array}{r}\text { Si2p } \\
\text { C1s } \\
\text { O1s } \\
\text { Ag3d }\end{array}$ & $\begin{array}{l}103.22 \\
285.83 \\
533.30 \\
369.17\end{array}$ & $\begin{array}{r}38795.39 \\
72742.92 \\
102850.76 \\
8781.50\end{array}$ & $\begin{array}{r}0.27 \\
0.25 \\
0.66 \\
5.2\end{array}$ & $\begin{array}{r}22.11 \\
47.68 \\
29.70 \\
0.52\end{array}$ \\
\hline $\begin{array}{l}\text { Ag-PDMS/Au } \\
\text { (Instant) }\end{array}$ & $\begin{array}{r}\mathrm{Si} 2 \mathrm{p} \\
\mathrm{C} 1 \mathrm{~s} \\
\mathrm{O} 1 \mathrm{~s} \\
\mathrm{Ag} 3 \mathrm{~d} \\
\mathrm{Au} 4 \mathrm{f} \\
\mathrm{Cl} 2 \mathrm{p}\end{array}$ & $\begin{array}{r}103.01 \\
199.33 \\
285.22 \\
368.80 \\
84.13 \\
199.33\end{array}$ & $\begin{array}{r}50115.90 \\
93443.80 \\
137722.34 \\
6543.61 \\
1202.60 \\
1230.61\end{array}$ & $\begin{array}{l}0.27 \\
0.25 \\
0.66 \\
0.25 \\
4.95 \\
0.26\end{array}$ & $\begin{array}{r}22.05 \\
47.20 \\
30.26 \\
0.27 \\
0.03 \\
0.19\end{array}$ \\
\hline $\begin{array}{l}\text { Ag-PDMS/Au } \\
(7 \mathrm{~h})\end{array}$ & $\begin{array}{l}\mathrm{Si} 2 \mathrm{p} \\
\mathrm{C} 1 \mathrm{~s} \\
\mathrm{O} 1 \mathrm{~s} \\
\mathrm{Ag} 3 \mathrm{~d} \\
\mathrm{Au} 4 \mathrm{f} \\
\mathrm{Cl} 2 \mathrm{p}\end{array}$ & $\begin{array}{r}102.97 \\
285.20 \\
533.03 \\
368.70 \\
85.21 \\
199.33\end{array}$ & $\begin{array}{r}51411.59 \\
94922.04 \\
136975.44 \\
4743.72 \\
2665.25\end{array}$ & $\begin{array}{l}0.27 \\
0.25 \\
0.66 \\
5.2 \\
4.95 \\
0.26\end{array}$ & $\begin{array}{r}22.4 \\
47.57 \\
29.77 \\
0.19 \\
0.07\end{array}$ \\
\hline
\end{tabular}

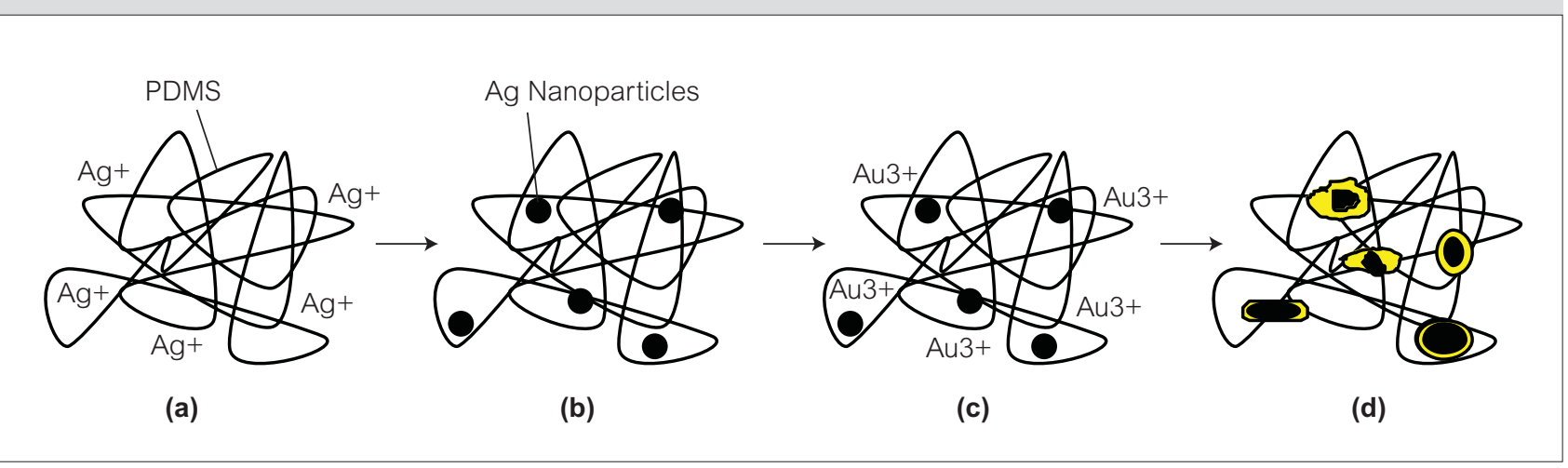

Schematic of the polymer-mediated galvanic replacement reaction (a) Silver ions ( $\mathrm{Ag}^{+}$) are reduced in the polymer matrix by curing agent; (b) Ag nanoparticles are embedded into the PDMS matrix and form the Ag-PDMS nanocomposite; (c) Ag nanoparticles reduce the incoming Au ${ }^{3+}$ from the solution and (d) Au nanoparticles grow on the silver template

Figure 7 shows a schematic of the polymer-mediated replacement of $\mathrm{Ag}$ by $\mathrm{Au}$. It has been shown that the adsorption of metal nanoparticles in polymers such as polystyrene and PVP is affected by their size and polydispersity and by the nature of their interaction with the surface. ${ }^{21}$ In the case of $\mathrm{Ag}$ nanoparticles embedded in PDMS, the interaction is strong as the polymer, through the curing agent, directly participates in the reduction reaction. The Ag nucleates and grows in the polymer matrix as shown in Figures 7a-c. Because of the strong nanoparticlePDMS interaction, it is reasonable to assume that the replacement reaction occurs between the $\mathrm{Ag}$ and
Au nanoparticles embedded in the PDMS surface layer, rather than between the $\mathrm{Ag}$ and $\mathrm{Au}$ alone, as in the case of solution. At low temperature, the Ag-PDMS nanocomposite is 'frozen' and, in spite of the lower diffusion rate of $\mathrm{Au}^{3+}$, a high concentration of core-shell particles will form, as seen in Figure 4. At this point, due to the proximity, the particleparticle interactions become stronger than the interaction of the particle with the polymer. When the quite fragile core-shell structures collapses, due to the high affinity between the gold particles, they aggregate and elongated domains are formed as seen in Figure 2b. This morphology is significantly 
different from that of Au-PDMS prepared directly from $\mathrm{HAuCl}_{4}$. The ability to control aggregation of nanoparticles would lead to new nanostructures with a host of potential applications including biosensing, catalysis, nanoscale electronics, etc.

\section{Conclusion}

The Ag/Au galvanic replacement reaction that takes place at the interface of PDMS/aqueous gold salt solution is investigated under various conditions and the results compared with that performed in an aqueous reaction environment. It has been found that the outcome of the replacement reaction, that is, the ribbon-like elongated Au aggregates, is significantly different from those reported in the literature. Core-shell nanoparticles as intermediate state were found when the replacement reaction was carried out at low temperature $\left(0^{\circ} \mathrm{C}\right)$. The collapse of the core-shell nanostructures well before the end of the replacement reaction is accounted for by their large size and the presence of a very thin Au shell as shown by both SEM and compositional surface analysis. Assembling metal nanoparticles into welldefined architectures will lead to properties distinctly different from the corresponding isolated particles.

\section{Acknowledgements}

This work was supported by a grant from NSERC, Canada and Canadian Commonwealth Graduate Study Exchange Programe.

\section{About the authors}

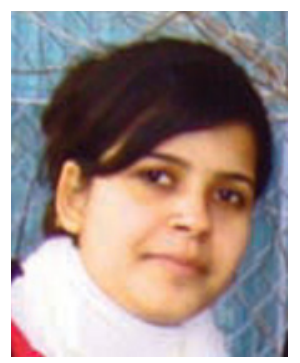

Pooja Devi is a Master student at Indian Institute of Technology (Roorkee), India, enrolled in M.Tech Nanotechnology Programme. She was selected in Canadian Commonwealth Graduate Study Common Wealth Scholarship program to work on her master dissertation at Concordia University in Montreal. She has reported the results of her first paper on "In situ synthesis and surface modification of Gold-Poly (dimethylsiloxane) nanocomposite for biosensing application" at BIOTECHNO-2010 International Conference at Cancun, Mexico. Currently, she is working as a Scientist at the Indian Government CSIR Laboratory, CSIO (Central Scientific Instruments Organization) in Chandigarh.

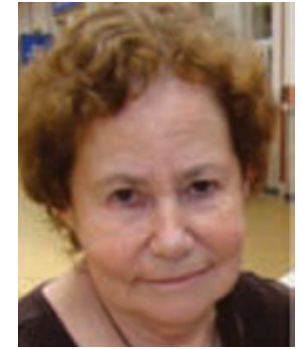

Dr. Simona Badilescu is a senior scientist with a background in physical chemistry and a rich experience in teaching and research. She received her Ph.D. degree from the University of Bucharest (Romania) and specialized in molecular spectroscopy, surface science and analytical applications of infrared spectroscopy. Dr. Badilescu

has several years of experience in an industrial environment as Director of the Spectroscopy Department of a Petrochemical Company in Romania. After three years of teaching in Algeria as Associate Professor at the University of Blida, she came with her family to Canada and was a Research Associate at the Universite de Montreal in the Chemistry Department. Her research interest focused on the vibrational spectroscopy of molecules of biological interest. Since 1987, she has been part of an interdisciplinary group, at the University of Moncton in New Brunswick, working on ATR spectroscopy of thin film systems. Afterward, she was a Professor of Chemistry at the University of Moncton in New Brunswick and later a Senior Scientist in the Physics Department. Dr. Badilescu joined the Electrical \&Computer Engineering Department in 2002 and since then, she was part of the Nanomaterials and Devices laboratory and contributed to different projects related to biosensing. Presently, she is part of the Nanomaterials and Nanodevices laboratory in the Mechanical Engineering Department. Dr. Badilescu published two books and several chapters, mainly on topics related to spectroscopy. She is author of more than 200 articles and conference papers. She is a member of several professional societies and a reviewer for Journals such as J. of Physical Chemistry, Applied Physics Letters, Advanced Materials, etc.

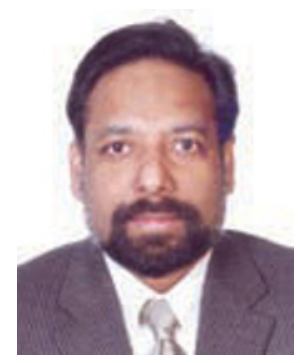

Dr. Muthukumaran Packirisamy is a Professor and Concordia Research Chair on Optical BioMEMS in the Department of Mechanical and Industrial Engineering, Concordia University, Canada. He is the recipient of Fellow and I.W.Smith award from Canadian society for Mechanical Engineers, Concordia University Research Fellow, Petro Canada Young Innovator Award and ENCS Young Research Achievement Award. His research interest includes Optical BioMEMS, Integration of Microsystems and Micro-Nano integration.

He obtained his Ph.D from Concordia University, Masters from Indian Institute of Technology Madras, India and Bachelors from University of Madras, India. He also worked for many MEMS industries in Canada. He is presently involved with developing BioMEMS devices in collaboration with industries. 
An author of more than 225 articles published in journals and conference proceedings, he has nine patents in the area of microsystems.

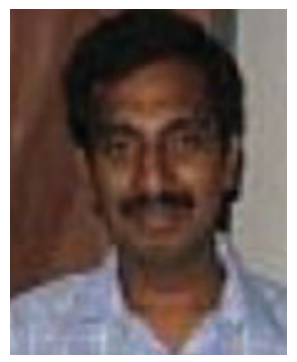

Dr. P. Jeevanandam received his PhD in Chemistry from Indian Institute of Science, Banglore, India in 1998. He was Post-Doctoral Fellow at Department of Chemistry, Bar-llan University, ISRAEL with Prof. Aharon Gedanken and, later on, he joined Kansas State University as a Research Associate with Prof. Ken Klabunde. Currently, he is an Assistant Professor in the Department of Chemistry, Indian Institute of Technology, Roorkee. He and his lab members are actively involved in topics such as: adsorbents based on nanostructured materials, nanostructured inorganic materials, nanorods of metal oxides and metal sulphides, optical properties of nanomaterials. He has published about 30 research articles, written three book chapters and has a patent as well.

\section{References}

1 C.J. Murphy, T.K. Sau, A.M. Gole, C.J. Orendorff, J. Gao, L. Gou, S.E. Hunyadi, T.Li, J. Phys. Chem. B, 2005, 109, 13857

2 Y. Ishihara, Shokubai Kasei Giho, 2002, 19, 82

3 D.A. Stuart, A.J. Haes, C.R. Yonzon, E.M. Hicks, R.P. Van Duyne, IEEE Proc. Nanobiotechnol 152, 13 (2005)

4 A.J. Haes, L. Chang, W.L. Klein, R.P. Van Duyne, J. Am. Chem. Soc, 2005, 127, 2264
5 S.E. Skrabalak, J. Chen, Y. Sun, X. Lu, L. Au, C.M. Cobley and Y. Xia, Acc. Chem. Res, 2008, 41, 1587

6 B.G. Prevo, S.A. Esakoff, A. Mikhailovsky and A. Zasadinski, Small, 2008, 4, 1183

7 X. Lu, J. Chen, S.E. Skrabalak and Y. Xia, Proc. IMechE, 2008, 221, 1

8 Y. Sun and Y. Xia, J. Am. Chem. Soc, 2004, 126, 3892

9 X. Lu, H.Y. Tuan, J. Chen, J.Y. Li, B. Korgel and Y. Xia, J. Am. Chem. Soc, 2007, 129, 1733

10 Q. Zhang, J.Y. Lee, J.Yang, C. Boothroyd and J. Zhang, Nanotechnology, 2007, 18, 245605

11 A. Pearson, A.P. O'Mullane, V. Bansal and S.K. Bhargava, Chem. Commun. 2010, 46, 731

12 Y.Z. Fu, X.D. Xiu, J.H. Liao and J.M. Wang, Journal of Dispersion Science and Technology, 2008, 29, 1291

13 Y.L. Mi, Y.N. Chan, D. Trau, P.B. Huang, E.Q. Chen, Polymer, 2006, 47, 5124

14 H.J. Bai, H.L. Gou, J.J. Xu and H.Y. Chen, Langmuir, 2010, 26, 2924

15 I.Wong and C.M. Ho, Microfluid Nanofluid, 2009, DOI 10.1007/s10404-009-0443-4 published online 18 April 2009

16 C.E. Hoppe, C. Rodriguez-Abreu, M. Lazzari, M.A. LópezQuintela and. D. Solans, phys. Stat.sol. (a) 2008, 205, 1455

17 D.I. Uhlenhaut, P. Smith, W. Caseri, Adv. Mater, 2006, 18, 1653

18 G. Carotenuto, G. La Peruta, L. Nicolais, Sensors and Actuators, 2006, B114, 1092

19 P. Devi, A.Y. Mahmoud, S. Badilescu, M. Packirisamy, P. Jeevanandam and Vo-Van Truong, BIOTECHNO Conference, 2010, Cancun

20 L. Au, X. Lu, Y. Xia, Adv. Mater, 2008, 20, 2517

21 V.M. Sukhov, O.V. Dementeva, M.E. Kartseva, V.M. Rudoy and V.A. Ogarev, Colloid Journal, 2004, 66, 482 